\title{
A combination of leaf rust resistance gene $L r 34$ and lesion mimic gene $l m$ significantly enhances adult plant resistance to Puccinia triticina in wheat
}

\author{
LI Tao ${ }^{1,2 *}$, BAI GuiHua ${ }^{3 *} \&$ GU ShiLiang ${ }^{1}$ \\ ${ }^{1}$ Jiangsu Provincial Key Laboratory of Crop Genetics and Physiology; Key Laboratory of Plant Functional Genomics of Ministry of \\ Education, Yangzhou University, Yangzhou 225009, China; \\ ${ }^{2}$ Department of Plant Pathology, Kansas State University, Manhattan, KS 66506, USA; \\ ${ }^{3}$ USDA-ARS Hard Winter Wheat Genetics Research Unit, Manhattan, KS 66506, USA
}

Received October 8, 2011; accepted November 28, 2011; published online March 8, 2012

\begin{abstract}
Leaf rust caused by Puccinia triticina is an economically-important disease in wheat worldwide. A combination of different types of resistance genes may significantly enhance rust resistance under rust-favorable conditions. To investigate the interactions between the rust resistance gene $L r 34$ and the lesion mimic gene $l m$ on $1 \mathrm{BL}$ in Ning 7840, a segregating $\mathrm{F}_{8-10}$ population of 180 recombinant inbred lines was developed from Ning 7840/Chokwang and evaluated for both lesion mimic expression and leaf rust response at the adult plant stage in a greenhouse. A major quantitative trait locus (QTL), derived from Sumai 3, was co-localized with $\operatorname{Lr} 34$ on chromosome 7D and explained $41.5 \%$ of phenotypic variations for rust severity and $22.1 \%$ for leaf tip necrosis (LTN). The presence of Lr34 was confirmed by Lr34-specific markers cssfrl and cssfr2 in Ning 7840 and Sumai 3. Unlike Lr34, Im conditioned a spontaneous lesion mimic phenotype and had a significant effect on reducing uredinial size, and a smaller effect on severity. Additive effects were observed between $l m$ and $L r 34$ for severity and LTN, and an epistatic effect was observed for infection type. Single marker analysis also identified several other QTL with minor effects on severity, infection type, or LTN.
\end{abstract}

Triticum aestivum, Puccinia triticina, lesion mimic, slow rusting gene $\mathrm{Lr} 34$, leaf rust resistance

Citation: $\quad$ Li T, Bai G H, Gu S L. A combination of leaf rust resistance gene $L r 34$ and lesion mimic gene $l m$ significantly enhances adult plant resistance to Puccinia triticina in wheat. Chin Sci Bull, 2012, 57: 2113-2119, doi: 10.1007/s11434-012-5001-x

Leaf rust, caused by Puccinia triticina Erikss., is an economically-important wheat disease worldwide. More than 60 leaf rust resistance genes and quantitative trait loci (QTL) have been described in wheat [1], most of which are racespecific. Race-specific resistance genes may stop the development of epidemics by limiting the initial inoculum or reproduction after infection [2]. Nevertheless, the resistance provided by these genes can be temporary because virulent races of the pathogen emerge or increase to overcome racespecific resistance. In contrast, race-nonspecific genes provide durable resistance to a broad spectrum of races and may function by slowing down the rate of infection development, thus slowing down the spread of disease in the field

*Corresponding authors (email: taoli@yzu.edu.cn; guihua.bai@ars.usda.gov)
[2].

In wheat, only a few leaf rust resistance genes function in a race-nonspecific manner, such as $\operatorname{Lr} 34$ [3,4] and $\operatorname{Lr} 46$ $[5,6]$, which are also called "slow rusting genes". Many cultivars, including Jupateco 73, Forno, Sunvale, and Chinese Spring have been reported to carry $\operatorname{Lr} 34$ since it was first described in cultivar Frontana in 1966 [7]. Lr34 confers a non-hypersensitive reaction (HR) type of resistance [6] and was recently cloned as an $\mathrm{ABC}$ transporter [8]. This gene remains durable, and virulence towards plants carrying Lr34 has not been observed to date [8]. Lr34 increases the latent period and reduces uredinial size and receptivity [4]. Although such resistance provides durable adult plant resistance, the effect on reducing rust damage is still dependent on environmental conditions and Lr34 alone cannot 
provide adequate protection from rust infection during a severe epidemic. A combination of Lr34 and other genes may be required to provide sufficient protection during severe rust epidemics.

Lesion mimic (LM) spontaneously occurs in plants and resembles HR-like phenotypes in the absence of pathogen infection [9]. Because plants with LM usually demonstrate enhanced resistance to a broad spectrum of pathogen races in a race-nonspecific manner, it is thought that $\mathrm{LM}$ is involved in plant defense signaling pathways [9-11]. In rice, LM mutants ebr3 [10,12], spl [13] and blm [14] provide race-nonspecific resistance to rice blast. In Arabidopsis, LM mutants $l s d l$ and acd showed enhanced resistance to the bacterial pathogen Pseudomonas syringae and oomycete Peronospora parasitica [15-17]. In wheat, both naturally-occurring [18] and chemical- or transgenic-induced LM have been reported [19-22]. These mutants express HR-like phenotypes in pathogen-free environments and exhibit enhanced resistance to biotrophic pathogens, including $P$. triticina.

Previous experiments indicated that wheat line Ning 7840 exhibits LM symptoms on the leaf blade after heading that resemble the typical wheat response to early leaf rust infection. A recessive gene, $l m$, on chromosome 1BL controlled LM expression, and lines with LM demonstrated a significantly lower infection response compared with a line without LM [18]. In the current study, investigations of resistance components in the recombinant inbred line (RIL) population from Ning 7840/Chokwang indicated that Ning 7840 also carries Lr34. The main objectives in this study were to elucidate the effects of HR-like $l m$ and non-HR Lr34 on adult plant response to leaf rust and to reveal additional chromosomal loci involved in rust resistance.

\section{Materials and methods}

\subsection{Plant materials and leaf rust evaluation}

A population of $180 \mathrm{~F}_{8-10}$ RIL was developed from the cross Ning 7840/Chokwang by single seed descent. Ning 7840 is a Chinese wheat breeding line that has adult plant leaf rust resistance, exhibits leaf tip necrosis (LTN), and expresses the LM trait after heading. The Korean wheat cultivar Chokwang does not demonstrate LM or LTN. The two parents and the resulting RIL were vernalized at $4^{\circ} \mathrm{C}$ in a growth chamber for 7 weeks and then transplanted into $10 \mathrm{~cm}$ $\times 10 \mathrm{~cm}$ plastic pots in a greenhouse at $17 \pm 2^{\circ} \mathrm{C}$ (night) and $22 \pm 5^{\circ} \mathrm{C}$ (day) with supplemental light for $12 \mathrm{~h}$. Experiments were arranged in a randomized complete block design with two replicates (pots) of five plants per replicate. The experiment was performed twice in the greenhouses at Kansas State University, Manhattan, KS, USA, in spring 2008 and 2009. LM was recorded as either presence or absence of LM symptoms on the flag leaf.

Flowering plants were inoculated with $P$. triticina (iso- late PRTUS55), which is virulent to both Ning7840 and Chokwang at the seedling stage, to evaluate adult plant leaf rust response in the RIL population. Ning 7840 showed high resistance to PRTUS55 and Chokwang showed moderate resistance at the adult stage. The inoculated plants were incubated in a mist chamber at $15^{\circ} \mathrm{C}$ with $100 \%$ relative humidity for $12 \mathrm{~h}$ and then moved to a greenhouse at $17 \pm 2{ }^{\circ} \mathrm{C}$ (night) and $22 \pm 5^{\circ} \mathrm{C}$ (day) with $12 \mathrm{~h}$ of supplemental light during the daytime. Rust symptoms were scored $14 \mathrm{~d}$ after inoculation for severity (percentage of infected flag leaf area) as described by Peterson et al. [23]. Infection type (IT) was scored following the protocol by Roelfs et al. [24], and the two modifiers "+" and "-" from McIntosh et al. [25] was also introduced to describe IT. The scale for IT was: 0, necrotic flecks; 1 , small uredinia surrounded by necrotic tissue; 2, small to medium uredinia surrounded by chlorotic or necrotic tissue; 3 , medium uredinia with or without chlorosis; 4, large uredinia without chlorosis; X, random distribution of variable sized uredinia on a single leaf; Y, variable sized uredinia with larger pustules at the leaf tip; Z, variable sized uredinia with larger pustules at the leaf base; +, pustules larger than average; and -, pustules smaller than average. Since $\mathrm{X}, \mathrm{Y}, \mathrm{Z},+$ and - are modifiers of IT and qualitative, IT ratings were converted to numerical scales for statistical and QTL analyses following the "maximum rule"; only the highest IT score was recorded for a single leaf. For example, if an IT score of a flag leaf was $3+4$, then 4 was recorded. Similarly, the modifiers X, Y, and Z were also digitalized. For the RIL carrying Lr34 that displayed Z patterns with a larger pustule size at the leaf base than at the leaf tip, only the IT at the base was scored; + or - was numerically transformed to $+/-0.25$. For example, an IT $3+$ was converted to 3.25 and $3-$ was converted to 2.75 . Consequently, all of the qualitative modifiers were converted to corresponding numerical values.

\subsection{Genotyping and data analysis}

Parents were initially screened using a genome-wide set of simple sequence repeat (SSR) markers with 12 markers per chromosome. Additional markers in chromosomal regions where markers had been correlated with rust resistance were screened. A total of 176 markers polymorphic between parents were used to genotype all RIL. DNA extraction and PCR amplification procedures followed Yu et al. [26]. Amplified PCR fragments were separated in an ABI 3730 DNA Analyzer (Applied Biosystems, Foster City, CA) and scored using GeneMarker 1.6 (Softgenetics Inc., State College, PA). Ambiguous data were removed following visuall inspection. Lr34-specific markers, cssfrl and cssfr2, which were designed based on a 3 bp polymorphism on exon 11 between Lr34 alleles, were amplified in Ning 7840 (Anhui 11/Aurora//Sumai 3) and its parents following the procedure described by Lagudah et al. [27]. Agarose gels (1\%) were used to resolve PCR products. 
Composite interval mapping (CIM) was performed with Windows QTLCartographer version 2.5 [28] using data from individual experiments and the means of two experiments. A LOD value of 3.0 was set as the threshold to claim the presence of a QTL. If the QTL was below the threshold in CIM, then a single marker regression model was applied to reveal associations between marker loci and severity or IT. Analysis of variance (ANOVA) was performed to evaluate differences in rust response between $l m$ and $L r 34$ alone and in combination. Statistical analysis was conducted using Matlab software (MathWorks Inc, Natick, Massachusetts, 2007).

\section{Results}

\subsection{Phenotypic variations of parents and RIL}

When inoculated with $P$. triticina (isolate PRTUS55), the mean percentage of infected leaf area was $15.6 \%$ for Ning 7840 (5.6\% in 2008 and $25.6 \%$ in 2009) but $40.0 \%$ for Chokwang (16.4\% in 2008 and $63.3 \%$ in 2009). A continuous distribution of severities within the RIL population, ranging from $2.0 \%$ to $82.5 \%$ (Figure 1). Transgressive segregation for increased resistance and susceptibility suggested that both Ning 7840 and Chokwang contributed different minor genes.

Ning 7840 produced an IT of $22+$, Chokwang 3+4, and the RIL had IT values ranging from $22+$ to 4 with a majority with 3 in both experiments. Most RIL with LM symptoms showed low IT $(\leqslant 22+)$, and an IT of $3+4$ was not observed in any RIL with LM. Most RIL without LM had high IT, except for six RIL (RIL 31, 60, 76, 80, 102 and 104) that had a consistent IT of $22+$ in both experiments. Average rust severity for RIL with LM was $27.2 \%$ while it was significantly $(P<0.0001)$ higher for non-LM RIL $(42.2 \%)$. RIL having both high IT $(3+4)$ and high severity $(>80.0 \%)$ always lacked LM.

\subsection{Chromosomal loci associated with leaf rust re- sistance in the Ning 7840/Chokwang population}

Ning 7840 showed LTN and a Z-pattern pustule distribution

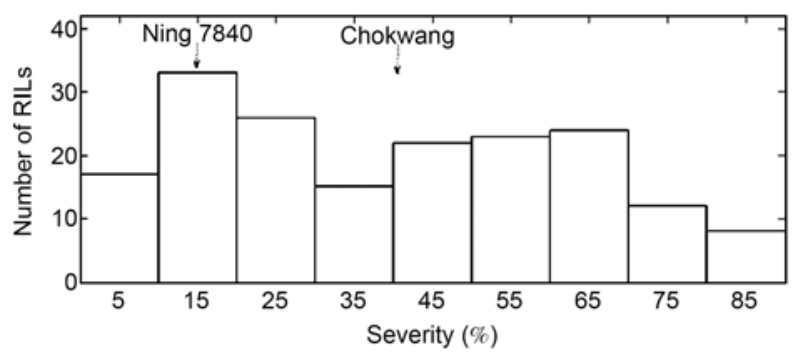

Figure 1 Frequency distribution of average leaf rust severity for the RIL population from Ning 7840/Chokwang in 2008 and 2009 experiments. with more and larger pustules at the leaf base and gradually decreasing pustule size toward the leaf tip in the adult plant stage. Both LTN and Z-pattern pustule distribution are the characteristics of the slow-rusting resistance gene Lr34. QTL mapping identified a major QTL responsible for both low rust severity and LTN on the short arm of chromosome 7D in the individual experiments in 2008 and 2009 as well as when using the means of the two experiments. This QTL was flanked by SSR markers Xgwm295 and Xbarc352 and its peak coincided with the marker $\operatorname{csLV34}$, which is a marker closely linked with Lr34 (Figure 2). The identified QTL explained $41.5 \%$ of the phenotypic variation for rust severity and $22.1 \%$ of the phenotypic variation for LTN.

Sumai 3 and Aurora were the parents of Ning 7840 (Anhui 11/Aurora//Sumai 3) and both showed LTN of different sizes, but only Sumai 3 showed a consistent Z-pattern pustule distribution in both experiments. PCR amplifications of Lr34-specific markers, cssfrl and cssfr2, generated a DNA fragment (517 bp) for cssfrl only with Ning 7840 and Sumai 3, while a PCR fragment (523 bp) for cssfr2 was produced only with Chokwang, Anhui 11, and Aurora. cssfrl is a positive control marker for $L r 34$, and produced a PCR fragment of 517 bp in wheat lines carrying Lr34, but generated no products in wheat lines lacking Lr34. cssfr2 is a negative control marker for Lr34 and produced a fragment of $523 \mathrm{bp}$ in wheat lines lacking Lr34 but generated no products in wheat lines carrying Lr34 [27], indicating that Lr34 in Ning 7840 was derived from Sumai 3.

In addition to $L r 34$ and $l m$, other genes with minor effects were involved in resistance to PRTUS55. Single marker analysis identified four additional markers associated with rust severity (Table 1). These additional markers were located on four chromosomes. Xsts3B-189 on 3BS and Xgwm3 on 3DS were positively associated with severity, and Ning 7840 contributed the favorable alleles. Markers Xgwm469 on 6DS and Xgwm361.2 on 7BS displayed negative associations with severity, suggesting Chokwang contributed alleles for rust resistance at these two loci.

Six additional markers were associated with IT. They were assigned to five chromosomes: 3AS, 3BS, 3DS, 6AS and 7BS (Table 1). Markers Xbarc323 on 3DS, Xsts3B-189 on 3BS, Xbarc3 on 6AS, and Xgwm43 on 7BS were positively associated with IT, suggesting that Ning 7840 contributed alleles associated with resistance. Markers Xbarc 321 on $3 \mathrm{AS}$ and $\mathrm{Xgwm} 361.2$ on 7BS were negatively associated with IT, suggesting that Chokwang carried alleles associated with resistance at these loci.

In addition to $L r 34$, three other loci were identified in Ning 7840 associated with LTN (Table 1). Xwmc710 on 4BS was negatively associated with the length of LTN $(P<$ $0.01)$, suggesting that the Ning7840 allele at this locus extended LTN. The other two markers, Xgwm539 on 2DL and Xgwm 361.2 on 7BS, were positively correlated to the length of LTN, suggesting Ning7840 alleles at these two loci reduced the length of LTN. 

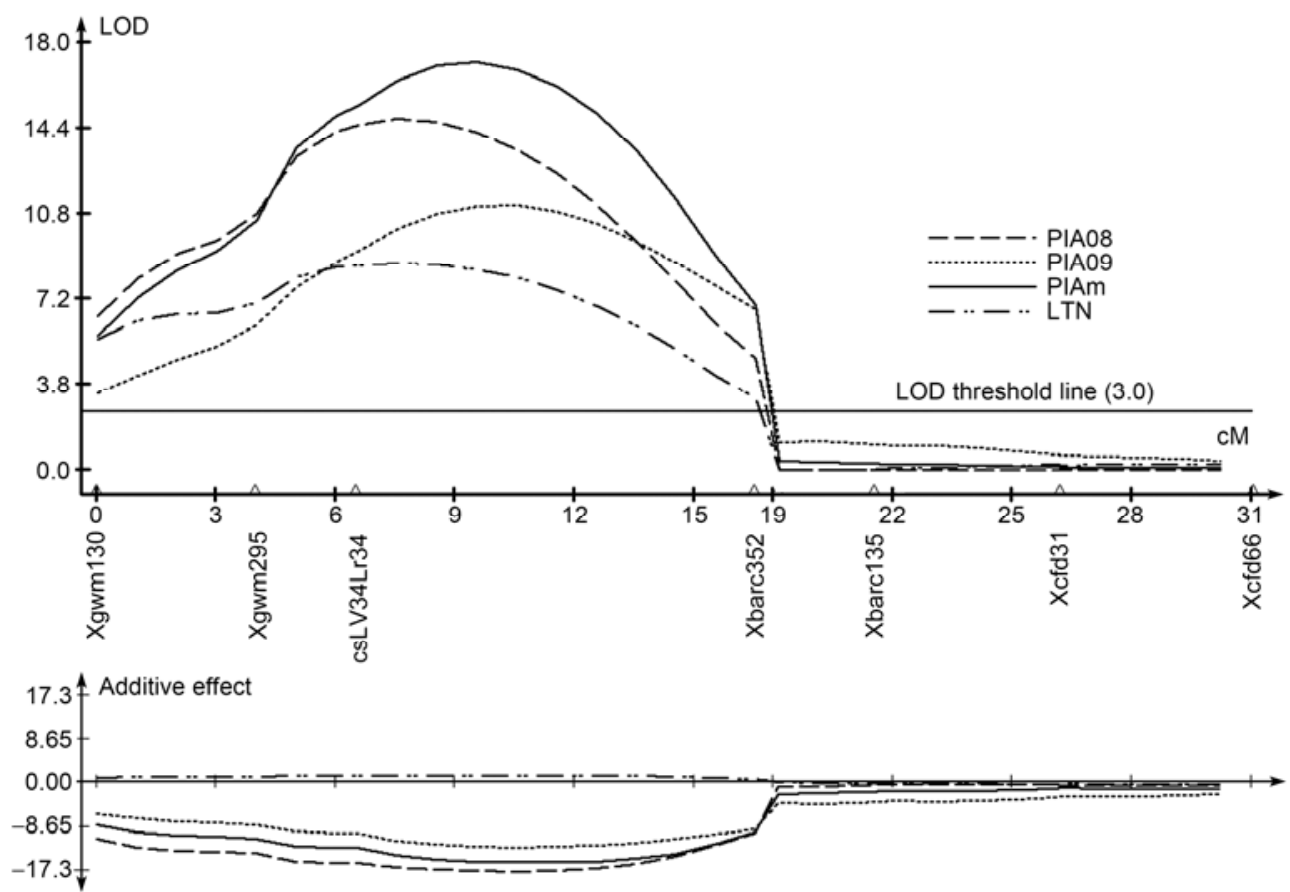

Figure 2 LOD curves for the Lr34 locus showing the QTL effect and location on chromosome 7D using percentages of infected flag leaf area (PIA) from 2008 (PIA08), 2009 (PIA09), the means over the two experiments (PIAm), and LTN data from 2009.

Table 1 Additional marker loci associated with severity, infection type (IT) and leaf tip necrosis (LTN) in the RIL population from Ning 7840/ Chokwang in 2008 and 2009 greenhouse experiments, as indicated by linear regression analysis using Matlab software at $\alpha=0.01$

\begin{tabular}{clcllc}
\hline Trait & \multicolumn{1}{c}{ Marker } & Effect & VE $^{\text {a) }}$ & $P$ value & Chromosome \\
\hline Severity & Xsts3B-189 & 4.408 & 0.029 & $<0.001$ & $3 \mathrm{BS}$ \\
& Xgwm469 & -4.13 & 0.029 & $<0.01$ & $6 \mathrm{DS}$ \\
& Xgwm361.2 & -4.08 & 0.028 & $<0.01$ & $7 \mathrm{BS}$ \\
& Xgwm3 & 3.983 & 0.027 & $<0.01$ & 3DS \\
IT & Xbarc323 & 0.102 & 0.05 & $<0.0001$ & 3DS \\
& Xbarc321 & -0.155 & 0.047 & $<0.0001$ & $3 \mathrm{AS}$ \\
& Xsts3B-189 & 0.139 & 0.039 & $<0.001$ & 3BS \\
& Xgwm361.2 & -0.224 & 0.038 & $<0.0001$ & 7BS \\
& Xbarc3 & 0.086 & 0.036 & $<0.0001$ & 6AS \\
& Xgwm43 & 0.19 & 0.03 & $<0.01$ & 7BS \\
LTN & Xwmc710 & -0.729 & 0.056 & $<0.0001$ & 4BS \\
& Xgwm539 & 0.705 & 0.05 & $<0.001$ & 2DL \\
& Xgwm361.2 & 0.603 & 0.039 & $<0.01$ & 7BS \\
\hline
\end{tabular}

a) Proportion of phenotypic variances explained by the marker.

\subsection{Relationship between $\mathrm{lm}$ and $\mathrm{Lr} 34$ in terms of rust response}

The LM phenotype had a marked effect on rust infection type and severity $(P<0.0001)$ and a marginally significant effect on LTN $(P=0.012)$ (Table 2$)$. The presence of $L r 34$ significantly reduced both severity and LTN $(P<0.0001)$, but not IT. The interaction between $\operatorname{lm}$ and $L r 34$ was mar- ginally significant for IT $(P<0.05)$, but not for severity and LTN. Since $l m$ and Lr34 have much larger effects, to analyze the interactions between $\operatorname{lm}$ and $L r 34$, the RIL were classified into four groups: $l m+L r 34+, l m+L r 34-, l m-L r 34+$, and $l m-L r 34-$. Variations in rust severity were significant among the four groups (Table 3). The two groups with Lr34 had significantly lower severities than the groups without Lr34. The group with both resistance alleles showed the lowest severity $(14.5 \%)$, and the group without any of the resistance alleles showed the highest severity (54.4\%). The group having Lr34 alone had significantly lower rust severity than the group having $l m$ alone, suggesting that $L r 34$ had a larger effect on the reduction of rust severity than $l m$, although both $l m$ and $L r 34$ played a role in reducing disease severity.

A significant difference existed between the groups with Im compared with those without the allele $(P<0.0001)$ (Table 3). RIL in the group with $l m$ had a smaller uredinial size than those in the group without $l m$, indicating that $l m$, rather than $\operatorname{Lr34}$, played a significant role in reducing IT in Ning 7840 .

The differences in the mean length of flag leaf tip necrosis were significant among the four groups $(P<0.001)$ (Table 3$)$. The group with both genes displayed the longest LTN, whereas the group without either exhibited the shortest LTN. On average, the presence of $l m$ alone increased LTN by $1.2 \mathrm{~cm}$, and the presence of $L r 34$ increased it by $2.1 \mathrm{~cm}$. $L r 34$ and $l m$ appeared to have an additive effect on LTN and intergenic interaction was not significant (Table 2, $P=0.512)$. 
Table 2 ANOVA analysis of the effects of $l m$ and Lr34 on severity, infection type (IT), and leaf tip necrosis (LTN) of RIL from Ning 7840/Chokwang in 2008 and 2009 greenhouse experiments

\begin{tabular}{|c|c|c|c|c|c|c|c|c|c|c|}
\hline & \multicolumn{5}{|c|}{ Severity } & \multicolumn{3}{|c|}{ IT } & \multicolumn{2}{|c|}{ LTN } \\
\hline & $D F^{\mathrm{a})}$ & $S S$ & $F$ & $P$ & $M S$ & $F$ & $P$ & $M S$ & $F$ & $P$ \\
\hline $\operatorname{lm}$ & 1 & 9974.542 & 28.402 & $2.989 \times 10^{-7}$ & 12.539 & 102.893 & $2.498 \times 10^{-19}$ & 48.636 & 6.433 & 0.012 \\
\hline Lr34 & 1 & 28707.217 & 81.742 & $2.778 \times 10^{-16}$ & 0.115 & 0.947 & 0.332 & 284.028 & 37.568 & $5.638 \times 10^{-9}$ \\
\hline $\operatorname{lm} \times \operatorname{Lr} 34$ & 1 & 434.812 & 1.238 & 0.267 & 0.560 & 4.596 & 0.033 & 3.264 & 0.432 & 0.512 \\
\hline Error & 176 & 351.194 & & & 0.122 & & & 7.560 & & \\
\hline
\end{tabular}

a) $D F$, degree of freedom; $S S$, sums of squares; $M S$, mean square; $F, F$ statistics; $P$, probability.

Table 3 Pair-wise comparisons of gene effects on rust severity, infection type (IT), leaf tip necrosis (LTN), and Z-pattern rust distribution on flag leaves among four $\mathrm{lm}$ and $\mathrm{Lr} 34$ gene combinations in a RIL population from Ning 7840/Chokwang in 2008 and 2009 greenhouse experiments

\begin{tabular}{cccccc}
\hline Group $^{\text {a) }}$ & $\begin{array}{c}\text { No. of } \\
\text { RIL }\end{array}$ & Severity $^{\mathrm{b})}$ & $\mathrm{IT}^{\mathrm{b})}$ & $\mathrm{LTN}^{\mathrm{b})}$ & $\begin{array}{c}\text { Z pat- } \\
\text { tern }^{\mathrm{c}}\end{array}$ \\
\hline$l m+L r 34+$ & 44 & $14.47 \pm 3.57 \mathrm{a}$ & $2.7 \pm 0.07 \mathrm{a}$ & $7.7 \pm 0.46 \mathrm{c}$ & + \\
$l m+L r 34-$ & 35 & $43.19 \pm 4.01 \mathrm{c}$ & $2.6 \pm 0.07 \mathrm{a}$ & $5.3 \pm 0.52 \mathrm{a}$ & - \\
$l m-L r 34+$ & 55 & $31.96 \pm 3.20 \mathrm{~b}$ & $3.1 \pm 0.06 \mathrm{~b}$ & $6.2 \pm 0.41 \mathrm{~b}$ & + \\
$l m-L r 34-$ & 46 & $54.43 \pm 3.49 \mathrm{~d}$ & $3.3 \pm 0.06 \mathrm{~b}$ & $4.1 \pm 0.45 \mathrm{a}$ & - \\
\hline
\end{tabular}

a) Gene combination in RIL. $l m+$, RIL with LM phenotype; $l m-$, RIL without LM phenotype; Lr34+, RIL carrying Lr34; and Lr34-, RIL without Lr34. b) Mean \pm standard error; $\mathrm{a}, \mathrm{b}, \mathrm{c}$, and d indicate significant differences between groups at $\operatorname{LSD}_{0.05}$. c) More and larger pustules occurred at the leaf base and gradually decreased in size toward the leaf tip in adult plants; wheat lines carrying Lr34 displayed a typical Z-pattern. +, presence of $\mathrm{Z}$ pattern; and -, absence of Z-pattern.

\section{Discussion}

Ning 7840 carries the non-HR resistance gene Lr34 and an HR-like resistance gene $l m$, and both conferred partial resistance to leaf rust in adult plants. Lr34 is widely recognized as reducing severity or receptivity as measured by the percentage of infected leaf area and accounts for 20\%-50\% of the phenotypic variation in various reports [4,29-33]. In this study, the effect of the rust resistance QTL at the Lr34 locus was consistent with previous reports [4,34]. In contrast, $I m$ increased rust resistance by reducing IT, a component different from that of Lr34. Thus, these two genes showed a complementary effect on overall rust resistance.

The Z-pattern pustule distribution on infected wheat leaves is a typical response of Lr34 and is characterized by more and larger pustules at the leaf base than at the leaf tip [4,34] (Figure S1(a)). Therefore, Lr34 should reduce IT, at least at the leaf tip. The insignificant association between Lr34 and IT in this study was due to the "maximum rule" used in the IT scoring system where IT is scored only at the leaf base, not at the tip. On average, RIL with both genes had a slightly higher IT than RIL with $l m$ alone and a slightly lower IT at the leaf base than RIL carrying Lr34 alone (Table 3). Therefore, the expression of $l m$ appeared to be negatively affected by expression of $\operatorname{Lr} 34$. This was also supported by the observation that LM symptoms appearing in the earlier stages of infection were reduced when LM was expressed in the presence of $L r 34$. This result could be due to the differences in the chronological expression patterns of the HR-like $I m$ and the non-HR Lr34. The effect of Lr34 was more pronounced at the grain filling stage than at other stages, and resistance at the flag leaf was most effective [4,8]. LM spots initially appeared around the heading stage [18]. Thus, the appearance of LM occurred earlier than the peak expression of Lr34 (i.e., at the grain filling stage) and the later expression of non-HR Lr34 might negatively affect Im expression, resulting in slightly higher IT for RIL with both genes than for RIL with $l m$ alone (Table 2).

Either Lr34 or $\mathrm{lm}$ alone may not be sufficient to control leaf rust under diverse climatic conditions. Singh et al. [35] reported that cultivars with $\operatorname{Lr34}$ alone showed approximately $40 \%$ of severity, but could reach unacceptably severe levels in epidemics. Results from this study suggest that Lr34 and $l m$ have different but complementary functions. The effect of Lr34 on severity reduction was superior to reductions in uredinial size, whereas $l m$ functioned in the opposite manner. Seventeen of 21 lines consistently showing low severity $(<10.0 \%)$ across the two experiments exhibited LM phenotype, LTN and Z-pattern pustule distribution, suggesting that a combination of race-nonspecific HRlike $I m$ and non-HR Lr34 may significantly improve adult plant resistance to a desirable level. It is commonly known that RIL with low IT do not always have low severity and vice versa. Only lines simultaneously having low IT and low severity were resistant in nature, and they usually carried both Lr34 and Im (Figure S1(c)). Our results indicate that a combination of the two distinct types of resistance genes could enhance the level of adult plant resistance. However, whether the expression of $l m$ has a negative impact on yield remains to be determined.

In addition to $L r 34$ and $l m$, the transgressive segregation of the RIL for severity (Figure 1) suggests that additional genes are involved in conferring resistance to PRTUS55. Single marker association analysis detected four and six additional markers that were significantly associated with severity and IT, respectively (Table 1). Xsts3B-189 on the short arm of chromosome $3 \mathrm{~B}$ of Ning 7840, previously associated with the fusarium head blight resistance gene Fhbl 
[36], was positively associated with both severity and IT, suggesting a gene on 3BS might also contribute to rust resistance. The marker Xgwm361.2 on 7BS chromosome was negatively associated with both severity and IT (Table 1). Thus, the associated locus in Ning 7840 might suppress the expression of leaf rust resistance to increase both severity and IT.

Results from this study indicated that Ning 7840 provided a unique source of resistance for improving adult plant leaf rust resistance in wheat. Resistance in Ning 7840 was mainly mediated by two distinct types of resistance genes (HR-like and non-HR) that conditioned complementary components of adult plant resistance. A combination of $L r 34$ and $l m$ resulted in reduced severity and smaller uredinial size.

We thank Robert Bowden (USDA-ARS Hard Winter Wheat Genetics Research Unit, Manhattan, KS 66506, USA) for providing the Puccinia triticina isolate used in this study. This work was partly funded by the Priority Academic Program Development of Jiangsu Higher Education Institution, the National Research Initiative of the USDA Cooperative State Research, Education and Extension Service, CAP (2006-55606-16629) and the Kansas Agricultural Experiment Station, Manhattan, Kansas, USA (10-325-J).

1 Bolton M D, Kolmer J A, Garvin D F. Wheat leaf rust caused by Puccinia triticina. Mol Plant Pathol, 2008, 9: 563-575

2 Gurr S J, Rushton P J. Engineering plants with increased disease resistance: What are we going to express? Trends Biotechnol, 2005, 23: $275-282$

3 Dyck P L. The association of a gene for leaf rust resistance with the chromosome 7D suppressor of stem rust resistance in common wheat. Genome, 1987, 29: 467-469

4 Singh R P, Huerta-Espino J. Effect of leaf rust resistance gene Lr34 on components of slow rusting at seven growth stages in wheat. Euphytica, 2003, 129: 371-376

5 Singh R P, Mujeeb-Kazi A, Huerta-Espino J. Lr46: A gene conferring slow-rusting resistance to leaf rust in wheat. Phytopathology, 1998, 88: 890-894

6 Martinez F, Niks R E, Singh R P, et al. Characterization of $L r 46$, a gene conferring partial resistance to wheat leaf rust. Hereditas, 2001, 135: 111-114

7 Dyck P, Amborski D, Anderson R. Inheritance of adult-plant leaf rust resistance derived from the common wheat varieties Exchange and Frontana. Can J Genet Cytol, 1966, 8: 665-671

8 Krattinger S G, Lagudah E S, Spielmeyer W, et al. A putative ABC transporter confers durable resistance to multiple fungal pathogens in wheat. Science, 2009, 323: 1360-1363

9 Lorrain S, Vailleau F, Balague C, et al. Lesion mimic mutants: Keys for deciphering cell death and defense pathways in plants? Trends Plant Sci, 2003, 8: 263-271

10 Campbell M A, Ronald P C. Characterization of four rice mutants with alterations in the defence response pathway. Mol Plant Pathol, 2005, 6: 11-21

11 Johal G S, Hulbert S H, Briggs S P. Disease lesion mimics of maize: A model for cell death in plants. BioEssays, 1995, 17: 685-692

12 Takahashi A, Kawasaki T, Henmi K, et al. Lesion mimic mutants of rice with alterations in early signaling events of defense. Plant $\mathrm{J}$, 1999, 17: 535-545

13 Yin Z C, Chen J, Zeng L R, et al. Characterizing rice lesion mimic mutants and identifying a mutant with broad-spectrum resistance to rice blast and bacterial blight. Mol Plant-Microbe Interact, 2000, 13: 869-876

14 Jung Y H, Lee J H, Agrawal G K, et al. The rice (Oryza sativa) blast lesion mimic mutant, blm, may confer resistance to blast pathogens by triggering multiple defense-associated signaling pathways. Plant Physiol Biochem, 2005, 43: 397-406

15 Greenberg J T, Ausubel F M. Arabidopsis mutants compromised for the control of cellular damage during pathogenesis and aging. Plant $\mathrm{J}$, 1993, 4: 327-341

16 Dietrich R, Delaney $\mathrm{T}$, Uknes $\mathrm{S}$, et al. Arabidopsis mutants simulating disease resistance response. Cell, 1994, 77: 565-577

17 Rate D N, Cuenca J V, Bowman G R, et al. The gain-of-function Arabidopsis acd6 mutant reveals novel regulation and function of the salicylic acid signaling pathway in controlling cell death, defenses, and cell growth. Plant Cell, 1999, 11: 1695-1708

$18 \mathrm{Li} \mathrm{T}$, Bai G. Lesion mimic associates with adult plant resistance to leaf rust infection in wheat. Theor Appl Genet, 2009, 119: 13-21

19 Boyd L, Minchin P. Wheat mutants showing altered adult plant disease resistance. Euphytica, 2001, 122: 361-368

20 Boyd L, Smith P, Wilson A, et al. Mutations in wheat showing altered field resistance to yellow and brown rust. Genome, 2002, 45: 1035-1040

21 Anand A, Schmelz E, Muthukrishnan S. Development of a lesionmimic phenotype in a transgenic wheat line overexpressing genes for pathogenesis-related (PR) proteins is dependent on salicylic acid concentration. Mol Plant Microbe Interact, 2003, 16: 916-925

22 Kamlofski C A, Antonelli E, Bender C, et al. A lesion-mimic mutant of wheat with enhanced resistance to leaf rust. Plant Pathol, 2007, 56: 46-54

23 Peterson R F, Campbell A B, Hannah A E. A diagrammatic scale for estimating rust intensity on leaves and stems of cereals. Can J For Res, 1948, 26: 496-500

24 Roelfs A P, Singh R P, Saari E E. Rust Diseases of Wheat: Concepts and Methods of Disease Management. Mexico, D.F.: CIMMYT, 1992

25 McIntosh R A, Wellings C R, Park R F. Wheat Rusts, an Atlas of Resistance Genes. East Melbourne: CSIRO Publications, 1995. 200

26 Yu J B, Bai G H, Cai S B, et al. Marker-assisted characterization of Asian wheat lines for resistance to Fusarium head blight. Theor Appl Genet, 2006, 113: 308-320

27 Lagudah E, Krattinger S, Herrera-Foessel S, et al. Gene-specific markers for the wheat gene $L r 34 / Y r 18 / P m 38$ which confers resistance to multiple fungal pathogens. Theor Appl Genet, 2009, 119: 889-898

28 Wang S, Basten C J, Zeng Z B. Windows QTL Cartographer 2.5. Department of Statistics, North Carolina State University, Raleigh, NC, 2007

29 Schnurbusch T, Paillard S, Schori A, et al. Dissection of quantitative and durable leaf rust resistance in Swiss winter wheat reveals a major resistance QTL in the Lr34 chromosomal region. Theor Appl Genet, 2004, 108: 477-484

30 Lagudah E S, McFadden H, Singh R P, et al. Molecular genetic characterization of the $L r 34 / Y r 18$ slow rusting resistance gene region in wheat. Theor Appl Genet, 2006, 114: 21-30

31 Kloppers F J, Pretorius Z A. Effects of combinations amongst genes Lr13, Lr34 and $L r 37$ on components of resistance in wheat to leaf rust. Plant Pathol, 1997, 46: 737-750

32 Rubiales D, Niks R E. Characterization of Lr34, a major gene conferring nonhypersensitive resistance to wheat leaf rust. Plant Dis, 1995, 79: 1208-1212

33 German S E, Kolmer J A. Effect of Gene Lr34 in the enhancement of resistance to leaf rust of wheat. Theor Appl Genet, 1992, 84: 97-105

34 Singh R P, Gupta A K. Expression of wheat leaf rust resistance gene Lr34 in seedlings and adult plants. Plant Dis, 1992, 76: 489-491

35 Singh R P, William H M, Huerta-Espino J, et al. Wheat rust in Asia: Meeting the challenges with old and new technologies. In: Proceedings of the 4th International Crop Science Congress. Brisbane, Australia. 2004

36 Liu S, Zhang X, Pumphrey M O, et al. Complex microcolinearity among wheat, rice, and barley revealed by fine mapping of the genomic region harboring a major QTL for resistance to Fusarium head blight in wheat. Funct Integr Genomics, 2006, 6: 83-89 
Open Access This article is distributed under the terms of the Creative Commons Attribution License which permits any use, distribution, and reproduction in any medium, provided the original author(s) and source are credited.

\section{Supporting Information}

Figure S1 Typical phenotypes of both $l m$ and $L r 34$ alone and in combination during PRTUS55 infection. (a) Typical phenotype of $L r 34$ with Z pattern; (b) susceptible line with high IT and high severity; (c) the RIL carrying Lr34 and $l m$ plus one or more genes with a highly reduced to almost immune phenotype; (d) typical phenotype of $l m$.

The supporting information is available online at csb.scichina.com and www.springerlink.com. The supporting materials are published as submitted, without typesetting or editing. The responsibility for scientific accuracy and content remains entirely with the authors. 